\title{
Photoactivated Nanoscale Temperature Gradient Detection Using X-ray Absorption Spectroscopy as a Direct Nanothermometry Method
}

Ana Espinosa,* German R. Castro, Javier Reguera, Carlo Castellano, Javier Castillo, Julio Camarero, Claire Wilhelm, Miguel Angel García, and Álvaro Muñoz-Noval*

Cite This: Nano Lett. 2021, 21, 769-777

Read Online

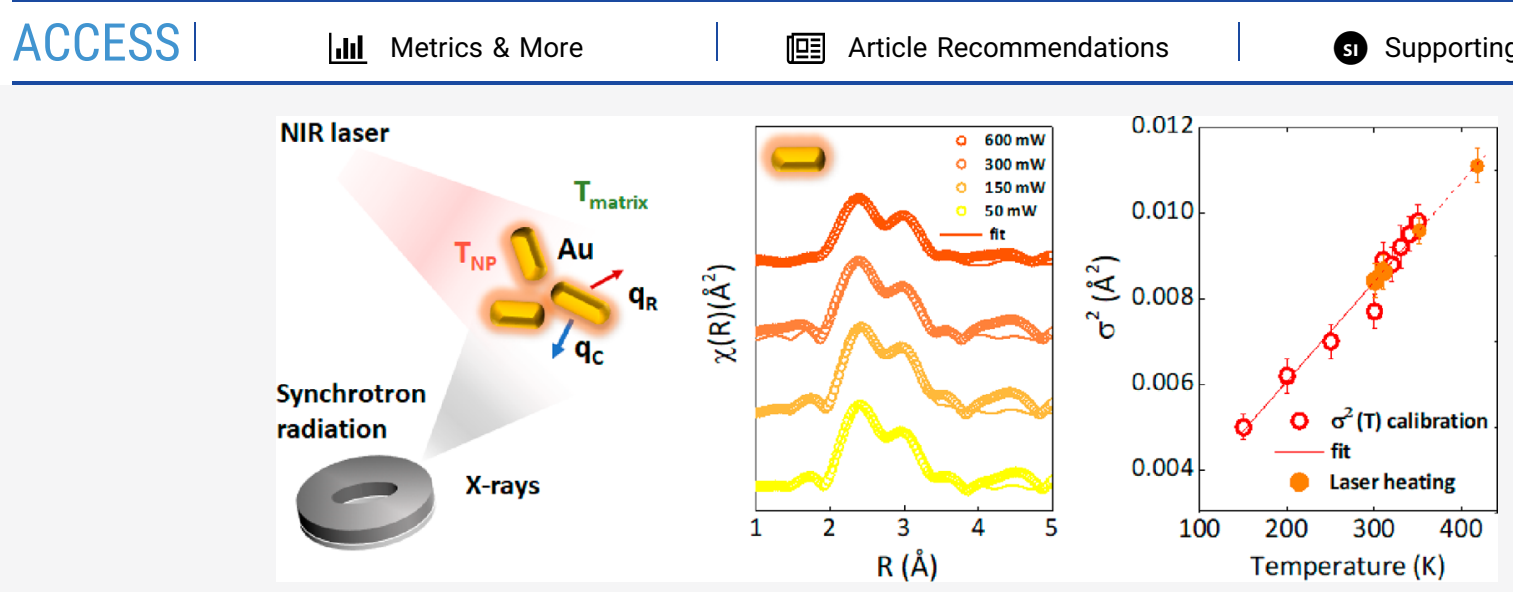

ABSTRACT: Nanoparticle-mediated thermal treatments have demonstrated high efficacy and versatility as a local anticancer strategy beyond traditional global hyperthermia. Nanoparticles act as heating generators that can trigger therapeutic responses at both the cell and tissue level. In some cases, treatment happens in the absence of a global temperature rise, damaging the tumor cells even more selectively than other nanotherapeutic strategies. The precise determination of the local temperature in the vicinity of such nanoheaters then stands at the heart of thermal approaches to better adjust the therapeutic thermal onset and reduce potential toxicity-related aspects. Herein, we describe an experimental procedure by X-ray absorption spectroscopy, which directly and accurately infers the local temperature of gold-based nanoparticles, single and hybrid nanocrystals, upon laser photoexcitation, revealing significant nanothermal gradients. Such nanothermometric methodology based on the temperature-dependency of atomic parameters of nanoparticles can be extended to any nanosystem upon remote hyperthermal conditions.

KEYWORDS: Nanothermometry, Photothermia, X-ray absorption spectroscopy, Nanothermal therapy, Plasmonic nanoparticles, Nanomedicine

$\mathrm{H}$ yperthermia therapy is an anticancer clinical practice based on elevation of the tumor temperature above physiological levels $\left(41-42{ }^{\circ} \mathrm{C}\right)$. It was essentially used as a potent cell killing sensitizer of other forms of tumor therapy, such as radiation therapy and chemotherapy, affecting multiple aspects of cellular metabolism (including DNA repair and synthesis of proteins). The use of nanoparticles (NPs), which can penetrate cell membranes and be remotely manipulated to induce local heating, has contributed to formulate more promising and safer approaches with increased therapeutic efficacy. The NPs can be externally thermoactivated by means of optical excitation (through the application of light in the near-infrared (NIR) range, photothermal therapy $(\mathrm{PTT})^{1}$ ) or magnetic stimulation (using alternating magnetic fields, magnetic hyperthermia $\left.(\mathrm{MHT})^{2}\right)$, depending on the type of nanostructure used (photothermal and/or magnetic nanoparticles).
Despite the high potential of nanoparticle-mediated hyperthermia, optimization of their heat capability in the tumor environment is still pursued through the fine-tuning of their nanostructure design, controlled aggregation, ${ }^{3,4}$ or chemical biostability. ${ }^{5}$ Moreover, the range of injected nanoparticle concentration was better delimited for an effective healing action for each thermal modality (PTT and MHT). ${ }^{6}$ However, therapeutic effects have also been observed by localized nanoparticle heating without causing a macroscopic temper-

Received: November 11, 2020

Revised: December 12, 2020

Published: December 31, 2020 
A

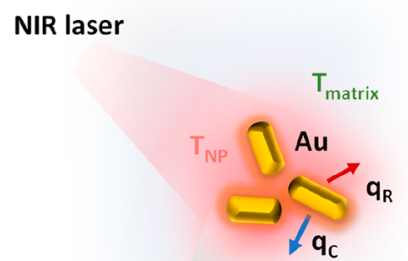

Synchrotron radiation

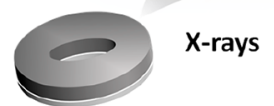

B

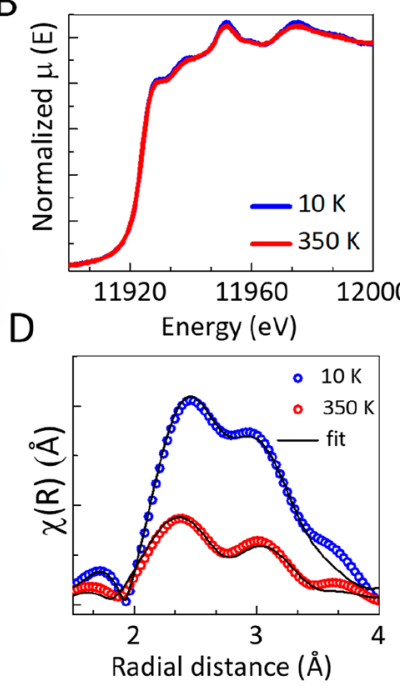

C

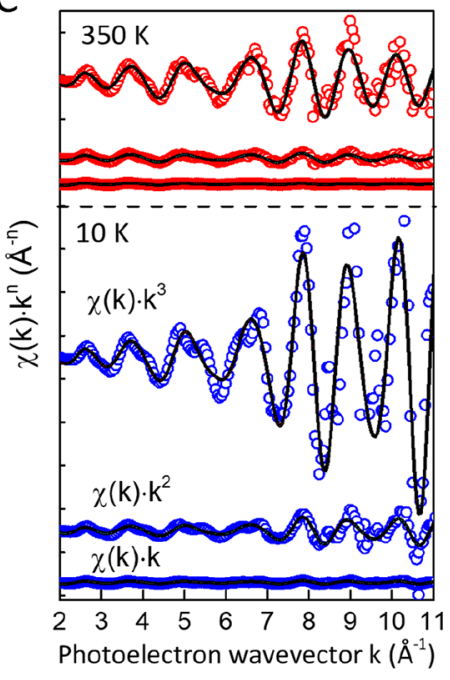

Figure 1. Correlation of the atomic structural and vibrational thermal parameters of gold nanomaterials obtained by X-ray absorption spectroscopy. (A) Scheme of photothermal excitation of Au nanoparticles (nanorods): the NP absorbs visible-NIR light resulting in a localized heating at the NP, leading to a conductive and a radiative heat flows; the temperature is higher than that of the surrounding medium. X-rays act as a probe of the local temperature in the nanomaterials. (B) XANES spectra at the Au $\mathrm{L}_{3}$-edge (11919 eV) of Au nanorods (AuNR) at 10 and $350 \mathrm{~K}$ obtained during the calibration curve measurements. (C) Fourier transform (FT, pair radial distribution) and fits of EXAFS spectra at $10 \mathrm{~K}$ (blue) and $350 \mathrm{~K}$ (red) of the AuNR. (D) EXAFS spectra and corresponding fits for the spectra weighted to $k, k^{2}$, and $k^{3}$.

ature increase. These "hot spots" can trigger effective cellular damage, induce remotely controlled drug release, or enable the activation of ion channels (TRPV1), which control the $\mathrm{Ca}^{2+}$ in cells and neurons. ${ }^{7,8}$ This soft and still effective hyperthermia has numerous advantages such as the limitation of nanoparticle toxicity and better preservation of neighboring healthy tissues.

Hence, there is a clear discrepancy between the temperature at the local vicinity of the nanomaterials and the temperature measured at the macroscopic scale in the surrounding medium, which requires an advance understanding. Nanoparticles are thermal foci where both dissipation and heat transmission at the nanoscale are mechanisms that differ from those that occur at the macroscale. Recently, some researchers have been involved in the study of nanometer temperature in nanoparticles under hyperthermia conditions. In most of the studies, this local temperature is accurately but indirectly determined by the use of an external element as luminescent optical labels ${ }^{9}$ (as $\mathrm{Ag}_{2} \mathrm{~S},{ }^{10} \mathrm{PbS}^{11}$ and $\mathrm{CdSe} / \mathrm{CdS} / \mathrm{ZnS}$ quantum dots), upconversion emitters $\left(\mathrm{Er}^{3+}, \mathrm{Yb}^{3+}, \mathrm{Nd}^{3+}\right.$, $\mathrm{Eu}^{3+}$, and/or $\mathrm{Tm}^{3+}$-doped nanomaterials $\left.{ }^{12-20}\right)$, thermosensitive polymers, ${ }^{21,22}$ or thermal-denaturation of DNA, ${ }^{23}$ which are attached to the heat nanogenerators activated by means of an alternating magnetic field or NIR-light. Direct measurements have been also proposed based on the analysis of thermal gradients generated in plasmonic systems which are only suitable for those systems which display such specific physical properties. ${ }^{24}$ In most cases, high thermal gradients have been reported at the nanoscale, where temperature elevation can be up to several times larger at the surface than in the long-range surrounding ambient. In this work, we use X-ray absorption fine structure spectroscopy (EXAFS) as a direct local temperature probe of nanoparticles under heat excitation. We evaluate the correlation of thermal vibration and structural parameters of state-of-the-art gold-based nanomaterials (as gold nanorods and star-like gold-branched nanohybrids) under NIR laser illumination.
Metallic NPs as gold or silver irradiated by a laser source of an adequate wavelength act as efficient heat sources that transfer energy to the surrounding medium. This effect is based on the localized surface plasmon resonance (LSPR) phenomenon, which produces strong enhancement and confinement of electric fields at the surface of nanoparticles. The LSPR band is tuned by the shape and size of the NP. Considering a spherical gold NP with radius $r_{\mathrm{NP}}$ immersed in a liquid or solid matrix at room temperature and light-irradiated at resonance, the conduction electrons are then excited by the local electric field, resulting in energy absorption and subsequent NP heating to the temperature $T_{\mathrm{NP}}$ determined by laser power, NP morphology, and dielectric medium, ${ }^{25-27}$ while the matrix initially remains at the environmental temperature $T_{\text {matrix }}$. For these NPs embedded in a liquid/solid matrix, the heat transfer is produced by two main mechanisms, radiation and conduction (see analysis in the Supporting Information). When dealing with nanoscopic objects, the heat conductive transport is no longer diffusive but ballistic in the immediate surroundings of the heat source, ${ }^{28,29}$ reducing the heat radiative efficiency with respect to conductive transport. Regarding the Au NPs, the ballistic heat transport therefore results in a less efficient heat exchange between the hot NP and the matrix, impairing the heat dissipation and increasing by orders of magnitude the thermal gradient around the NP.

\section{EXTENDED X-RAY ABSORPTION FINE STRUCTURE SPECTROSCOPY AS AN IN SITU PROBE TO CORRELATE THE LOCAL ATOMIC STRUCTURAL AND VIBRATIONAL THERMAL PARAMETERS}

The X-ray absorption spectroscopy (XAS) is a technique performed in synchrotron sources with element-specificity and high sensitivity, which provides precise information about the chemical state, coordination, and local structure for a particular element (Figure 1A). A XAS spectrum has two main parts: the $\mathrm{X}$-ray absorption near edge structure (XANES) (Figure 1B), and the extended X-ray absorption fine structure (EXAFS, 
Figures 1C and 1D) regions. The local structure of an element in a sample can be determined by analyzing the EXAFS. Through the Fourier analysis of the oscillatory spectrum of the photoexcited electron that interacts with the atomic local environment of the scattered atoms, it is possible to quantify structural and vibrational local parameters for a given element (Figure 1C). This can be performed by fitting the experimental spectrum to a structural model by means of the EXAFS equation, which contains the main local structure parameters:

$$
\chi(k)=\sum_{i} \frac{\left(N_{i} S_{0}^{2}\right) F_{i}(k)}{k R_{i}^{2}} \sin \left[2 k R_{i}+\delta_{i}(k)\right] e^{-2 \sigma_{i}^{2} k^{2}} e^{-2 R_{i} / \lambda(k)}
$$

The EXAFS equation is related to the number of atoms in each atomic shell around the scattered atom $\left(N_{i}\right)$, the radii of each shell $\left(R_{i}\right)$, and the variance of the radii of the surrounding atomic shell $\left(\sigma_{i}^{2}\right)$. This latter parameter, the Debye-Waller factor, is defined for each atomic shell surrounding the scattering atom and contains two contributions: (i) one related to the intrinsic dispersion of atomic distances due to structural disorder $\left(\sigma_{\mathrm{S}}^{2}\right)$ (a static term in absence of phase transitions) and (ii) a vibrational contribution related to the thermal movement of atoms $\left(\sigma_{\mathrm{T}}^{2}\right)$ that is intrinsically temperaturedependent. $^{30}$ Therefore, it can be expressed as

$$
\sigma^{2}(T)=\sigma_{\mathrm{S}}^{2}+\sigma_{\mathrm{T}}^{2}(T)
$$

The $\sigma_{\mathrm{T}}^{2}$ term is related to phonon oscillation and can be modeled following a Debye-Waller model for harmonic oscillations in a lattice. ${ }^{31}$ This term is usually very well described using the equation given by a correlated Debye model: ${ }^{31}$

$$
\sigma_{\mathrm{T}}^{2}=\frac{3 \hbar^{2}}{M k_{\mathrm{B}} \theta_{\mathrm{D}}}\left[\frac{1}{4}+\left(\frac{T}{\theta_{\mathrm{D}}}\right)^{2} \int_{0}^{\theta_{\mathrm{D}} / T} \mathrm{~d} x \frac{x}{e^{x}-1}\right]
$$

with $M$ being the mass of the scattering atom, $k_{\mathrm{B}}$ the Boltzmann constant, and $\theta_{\mathrm{D}}$ the Debye-Einstein temperature, which accounts for the bond strength. Eq 3 is in most cases a correct approximation for all phonon modes.

The $\sigma^{2}$ vs $T$ does not follow a linear response in the whole temperature range, as eq 3 shows. However, in the range of high $T(T \geq \theta)$, the $\sigma^{2}$ vs $T$ behaves linearly. ${ }^{32}$ This equation can be expanded in Taylor series considering $\theta / T<1$ and conserving only the higher order terms, which for the limit $\left(\theta_{\mathrm{D}} / T\right)^{2} \ll 1$ can be approximated to a linear equation:

$$
\sigma_{\mathrm{T}}^{2} \sim \frac{A}{\theta_{\mathrm{D}}}\left(\frac{1}{4}+\frac{T}{\theta_{\mathrm{D}}}\right)
$$

Therefore, $\sigma^{2}$ increases linearly with $T$ for sufficiently high temperatures. Because the Debye temperature of nanostructured and bulk Au in most cases is quite low, ${ }^{32}$ we can assume a linear behavior in the range of temperatures of hyperthermia conditions. Eq 4 provides an immediate way to obtain the NP temperature from given $\sigma^{2}$ value once the experimental conditions and the $\sigma^{2}(T)$ curve is obtained.

In a temperature-dependent experiment, considering a controlled range of temperatures where phase transitions do not occur, the number of atoms in each shell can be assumed to be constant, and only the radii $(R)$ and the vibrational variance $\left(\sigma_{\mathrm{T}}^{2}\right)$ will change with temperature. Precision limits of the EXAFS technique have been pushed to femtometer limits nowadays using cutting-edge instruments and data analysis, which is perfectly suitable for temperature-dependent experiments. ${ }^{33-35}$ In this nanothermometry application, once the calibration curve is obtained for a set of temperatures, this $\sigma^{2}(T)$ curve allows determination of the temperature values raised in the system under different laser excitation conditions.

\section{EXAFS ATOMIC PARAMETERS OF GOLD-BASED NANOPARTICLES AS A FUNCTION OF A WIDE RANGE OF TEMPERATURE VALUES}

The thermal dependence of both atomic parameters was studied in plasmonic gold-based nanoparticles. We selected single gold nanoparticles (nanorods AuNR (with an aspect ratio of $\approx 3.5(41 \pm 4 \times 11 \pm 2 \mathrm{~nm}))$ and hybrid star-like goldbranched nanoparticles (AuNS, in combination with an $20 \mathrm{~nm}$ iron oxide-core forming a Janus configuration $[\mathrm{Au}] /[\mathrm{Fe}]=2.3$, $35 \pm 5 \mathrm{~nm}$ of diameter). Both have been proven as excellent PTT agents when irradiated at the NIR range. ${ }^{6,36}$ They were synthesized using the chemical methods previously reported. $^{6,36,37}$ Transmission electron microscopy (TEM) images are illustrated in Figure 2. Both display LSPR bands
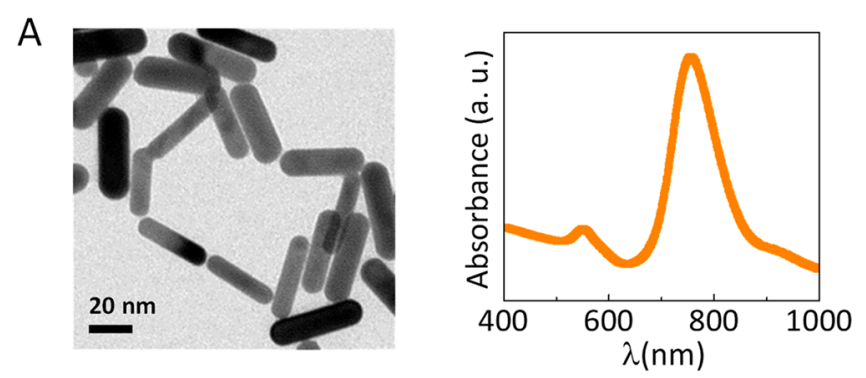

B
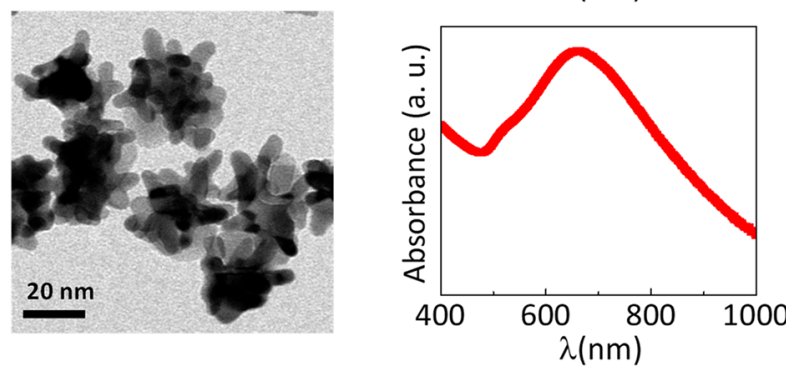

Figure 2. Two candidates of plasmonic nanoparticles used in photoinduced thermal therapy: single and hybrid gold-based nanomaterials. TEM images and UV-vis-NIR spectra of (A) AuNR and (B) AuNS.

in the NIR, centered at 600-900 nm (Figures 2A and B), with large extinction coefficients, ${ }^{6,36}$ which allow them to be lightinduced thermally activated with $808 \mathrm{~nm}$ laser wavelength.

An initial calibration was first performed to obtain the temperature dependence of the EXAFS Debye-Waller factors of gold-based nanoparticles in an extend range of temperatures. XAS measurements were taken at the $\mathrm{Au}_{3}$-edge $(11919 \mathrm{eV})$ in the CRG BM25A SpLine at the European Radiation Synchrotron Facilities (Grenoble, France). In this case, we initially selected the AuNR embedded in an X-ray transparent material (boron nitride (BN)) and measured in transmission mode. AuNR were mixed uniformly in a BN matrix and

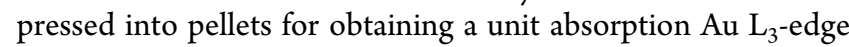
step jump. The samples were introduced into an LHe cryostat and thermalized in a gas atmosphere for a sufficiently long time to ensure that the temperature of the sample corresponds to 
the temperature of the bath. XANES spectra of AuNR are shown in Figure 1B at low and high temperature values of 10 and $350 \mathrm{~K}$. XANES profiles confirmed that $\mathrm{Au}$ is in its metallic crystallographic phase and in agreement with previous characterization. ${ }^{6}$ The EXAFS signal $\chi(k)$ was then extracted and weighted by a factor of $k^{2}$ over the $k$-range $\left(0-14 \AA^{-1}\right)$. Subsequently, the measurements were carried out in a large temperature range with multiple temperature values probed to get a precise interpolation from 10 to $350 \mathrm{~K}$ with steps between 10 and $50 \mathrm{~K}$, depending on the temperature region. As the sample is thermally activated from 10 to $350 \mathrm{~K}$, the EXAFS amplitude decreases systematically due to increased thermal motion of the gold atoms in the lattice, and therefore $\sigma_{\mathrm{T}}^{2}$, as eq 4 shows. In Figure $3 \mathrm{~A}$, the temperature-dependency
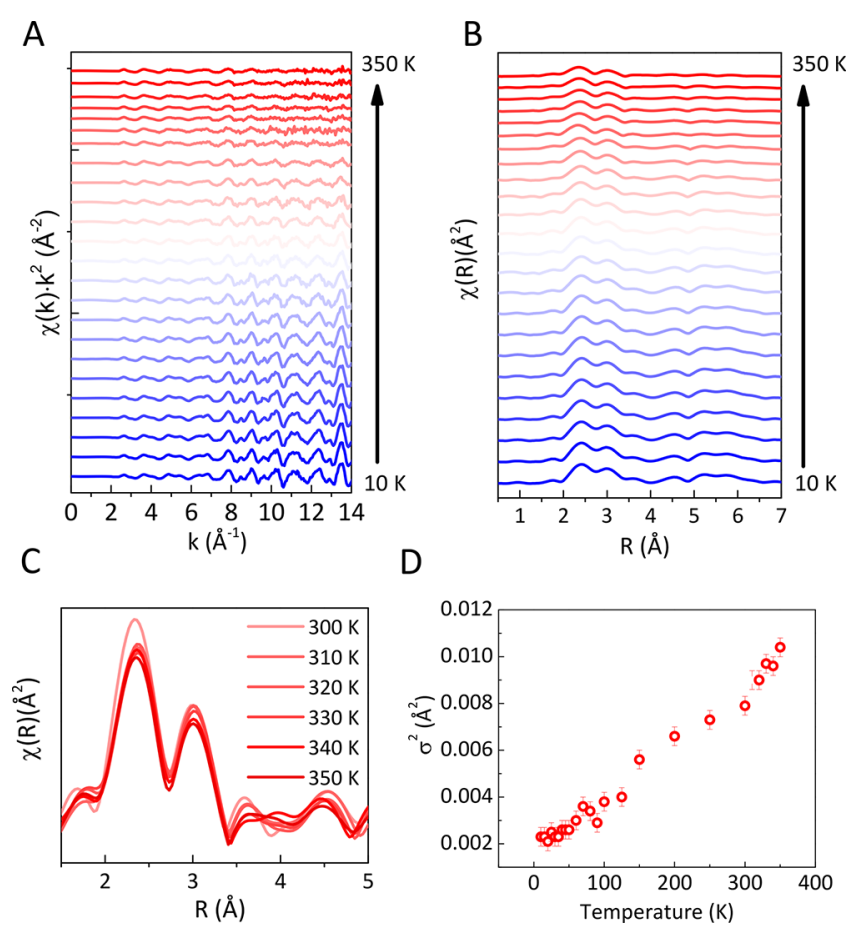

Figure 3. Thermal calibration of structural parameters of AuNR from temperature-dependent EXAFS measurements. (A) EXAFS spectra and (B) FT of the EXAFS signal from 10 to $350 \mathrm{~K}$. (C) FT of the EXAFS signal in the range of calibration from 300 to $350 \mathrm{~K}$. (D) Debye-Waller factor $\left(\sigma^{2}\right)$ of AuNR as a function of temperature obtained through the fits to the experimental EXAFS data, both independent of the Einstein model.

of the EXAFS spectra is displayed. The corresponding Fourier transform (FT) of EXAFS signal provides a pair radial distribution function of neighboring atoms in the lattice that contain information about its static and vibrational disorder (from 10 to $350 \mathrm{~K}$, Figure 3B). The FT reveals two main peaks corresponding to the $\mathrm{Au}-\mathrm{Au}$ bond distances. A short-range of temperatures above $300 \mathrm{~K}$ is shown in Figure 3C. As the temperature is increased, the intensities of the FT peaks decrease due to the increment of thermal activation and dynamic disorder. Because of the nanometer size of the AuNR, the corresponding FT is fitted to a Au fcc structure considering only the first atomic shell ${ }^{38}$ (for a nanocrystalline sample, the particle is so small that the neighboring atoms are reduced). The number of atomic neighbors $(N)$ and the nonstructural parameter $\left(E_{0}\right)$ were determined for the lowest temperature measurements (where the EXAFS signal is better defined) and constrained during subsequent fittings. The fitting parameters correspond then to the first atomic shell distance $(R)$ of the $\mathrm{Au}$ $\left(R_{\mathrm{Au}-\mathrm{Au}}=2.86 \AA\right.$ and coordination number $\left.N=12\right)$ and of its shell distance variance $\left(\sigma^{2}\right)$.

The calculated $\sigma^{2}$ for the AuNR along with the values obtained by fitting the experimental EXAFS data at multiple temperatures are shown in Figure 3D. The curve follows a linear tendency at higher temperatures, but it is sloped within the low-temperature region, as expected. ${ }^{32,39}$ A calibration of temperature-dependent structural parameters was also performed for the AuNS under the same conditions. The results are shown in Figure S1 of the Supporting Information.

\section{LOCAL TEMPERATURE MEASUREMENT OF NEAR-INFRARED PHOTOEXCITED HEATING OF PLASMONIC GOLD-BASED NANOPARTICLES USING EXAFS}

The accuracy of this method has been now tested in operando in both AuNR and AuNS systems upon NIR laser exposure. The experimental setup indeed allows measuring in situ and in real-time the generated heating of laser-illuminated nanoparticles simultaneously with the XAS. The samples were irradiated by employing an NIR laser $(808 \mathrm{~nm})$ at controlled increased irradiation power (from 0.05 to $0.6 \mathrm{~W}$ ). In the first set of experiments, each system was laser-irradiated for more than $1 \mathrm{~h}$ to ensure that the thermal equilibrium (or steady-state temperature) was reached. The averaged spectra were analyzed, and the Debye-Waller factor value $\sigma^{2}$ was determined for each irradiation condition and gold nanosystem, as presented in Figures 4A, B and D, E, respectively, and in Table S1. The interpolated $T$ value is then obtained for AuNR and AuNS (Figures 4C and F). As both figures show, increasing laser power results in increasing of the AuNR temperatures, and this relation is linear in the selected range of temperatures $(150-350 \mathrm{~K})$. The atomic length $(R)$ also increases with irradiation power for both gold nanosystems (see Table S1 and Figure S2).

For AuNR, the temperatures attained under these conditions are $314,332,357$, and $418 \mathrm{~K}$, corresponding to temperature increments $(\Delta T)$ of $19,37,62$, and $123{ }^{\circ} \mathrm{C}$ (see Table S1). For AuNS, the inferred temperature values were $325,336,356$, and $400 \mathrm{~K}$, corresponding to temperature increments $(\Delta T)$ of 31 , 42, 62, and $106{ }^{\circ} \mathrm{C}$ (see also Table S1). Remarkably, the temperature values reached by NPs in both cases are very high, and this was at low to intermediate laser powers. This behavior makes the laser power density and temperature relation diverge from a linear association at the nanoscale. This effect is even more pronounced in AuNS due to the association of iron oxide that, in one hand, acts as a thermal reservoir of the induced heat on plasmonic gold, and on the other hand, because the iron oxide (magnetite) also absorbs NIR light and releases heat to a lesser extent than that produced by the LSPR phenomenon of $\mathrm{Au}$ nanoparticles. ${ }^{6,40}$ This procedure provides precise temperature determination in the NPs with uncertainties below $5^{\circ}$ in all cases.

\section{COMPARISON WITH THE MACROSCOPIC TEMPERATURE MEASURED UNDER LASER IRRADIATION}

The values of the local temperature of AuNR and AuNS upon NIR laser-induced exposure measured by previous EXAFS measurements were compared with the global temperatures 
A

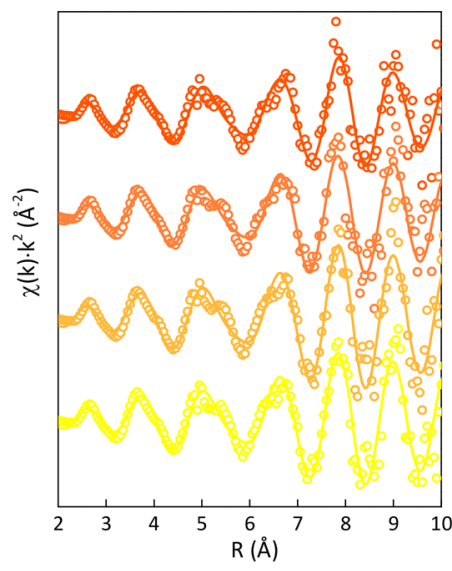

D

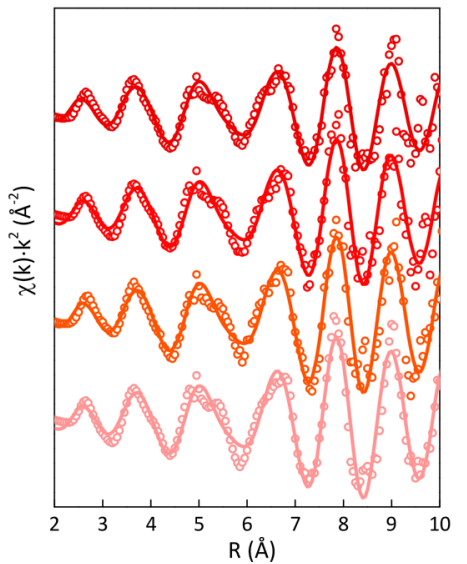

B

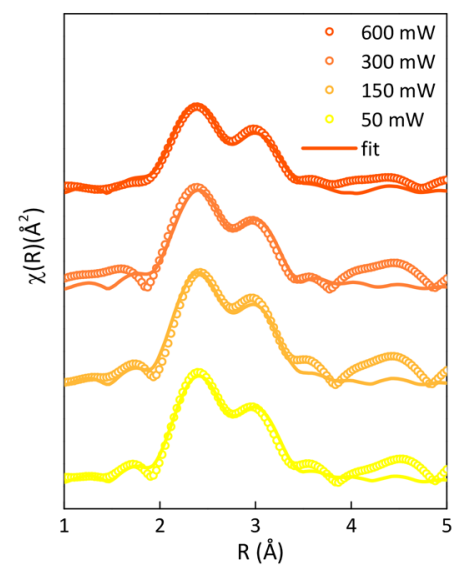

$\mathrm{E}$

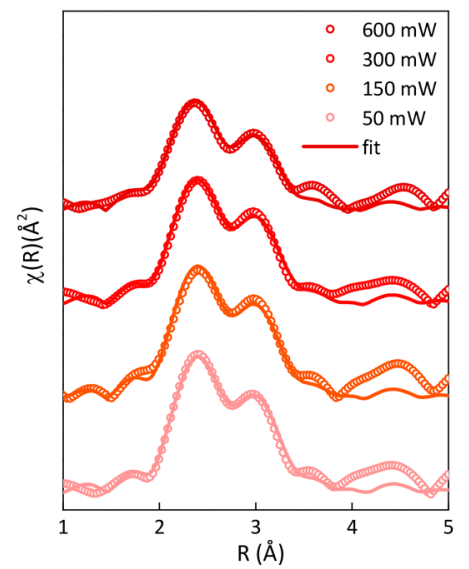

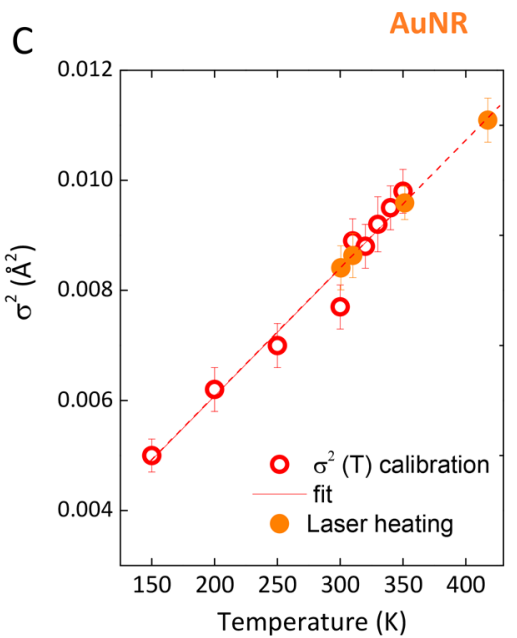

AuNS

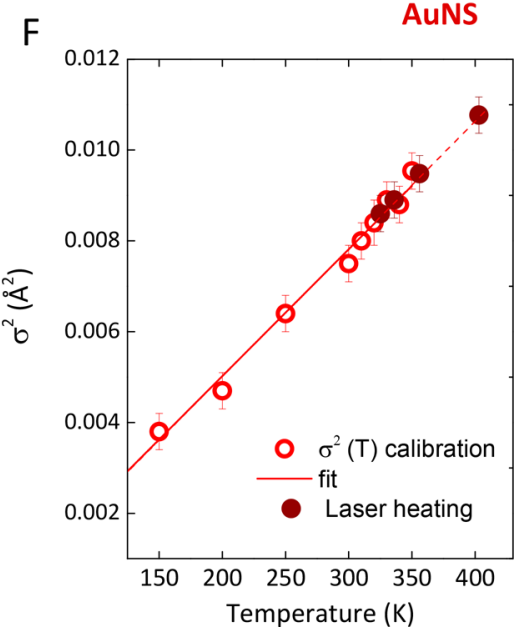

Figure 4. Local temperature measurement of the photoinduced heating in single and hybrid gold-based nanomaterials using EXAFS. (A, D) Experimental $k^{2}$-weighted EXAFS spectra and (B, E) the corresponding Fourier transforms of AuNR and AuNS under NIR laser at different density powers $(0.05-0.6 \mathrm{~W})$. (C, F) Debye-Waller factor parameters $\left(\sigma^{2}\right)$ of AuNR and AuNS as a function of temperature. The open symbols were obtained through the experimental EXAFS spectra calibration fits, and the solid symbols were calculated from the NIR laser excited nanomaterial EXAFS spectra fits. The solid lines show the fits based upon the Debye-Einstein model in the linear approximation (150-350 K).

determined using an IR thermographic camera under the exact same experimental conditions and setup configuration as the XAS measurements. Further, we used two configurations of the IR camera to observe the samples and the thermal distribution within. The IR camera then detected the average temperature of (i) the front-illuminated surface of the sample (central point) and of (ii) the sample profile at the transmission direction, which is the direction of the probed sample by $\mathrm{X}$ rays (experimental configuration shown in Figure 5A and B, respectively). These two IR camera readouts provide a noncontact and noninvasive temperature monitoring method suitable for photothermal studies. It also avoids the use of thermal probes which may incur in possible self-heating effects under laser illumination. The $\Delta T$ values reached under photoexcitation after $15 \mathrm{~min}$ of treatment at both configurations and the same laser power (0.05-0.6 W) for AuNR and AuNS are displayed in Figure 5C and D, respectively. In general, the EXAFS method points out higher thermal gradients. Figure 5E shows IR images of the heating for AuNR nanoparticles after $15 \mathrm{~min}$ under $0.6 \mathrm{~W}$ laser power at the two spatial experimental configurations (point and bars indicate the positions of the maximum and average reading temperature of the thermal mapping, respectively).

The AuNR at the lowest laser power $(0.05 \mathrm{~W})$ reaches $\Delta T=$ $4{ }^{\circ} \mathrm{C}$ as the maximum value obtained at the transmission mode that is remarkably lower than the one obtained from EXAFS parameters, which yielded $\Delta T=19^{\circ} \mathrm{C}$. This marked difference in heating performance is also evidenced at increasing laser powers until $0.6 \mathrm{~W}$, where laser illumination at $0.3 \mathrm{~W}$ generates a macroscopic temperature elevation of $\Delta T=53{ }^{\circ} \mathrm{C}$ compared with $\Delta T=62{ }^{\circ} \mathrm{C}$ from EXAFS analysis. Overall, at the local stage, the generated temperature at high laser density powers is around 1.5-fold higher than the one recorded with the IR camera at the transmission mode, while at lowest power, this value can overtake by 3 -fold the macroscopic reading.

The same thermal response tendency is observed by looking at the AuNS; however, in this case, the thermal gradient effect is even more noticeable, where the mean temperature is $1.5-2$ times lower than the above direct thermal evaluation (i.e., for $0.3 \mathrm{~W}$ laser power, $\Delta T=62{ }^{\circ} \mathrm{C}$ (EXAFS method) vs $\Delta T=44$ and $35{ }^{\circ} \mathrm{C}$ (global temperature at the surface and profile section of the sample, respectively)). However, this increased 

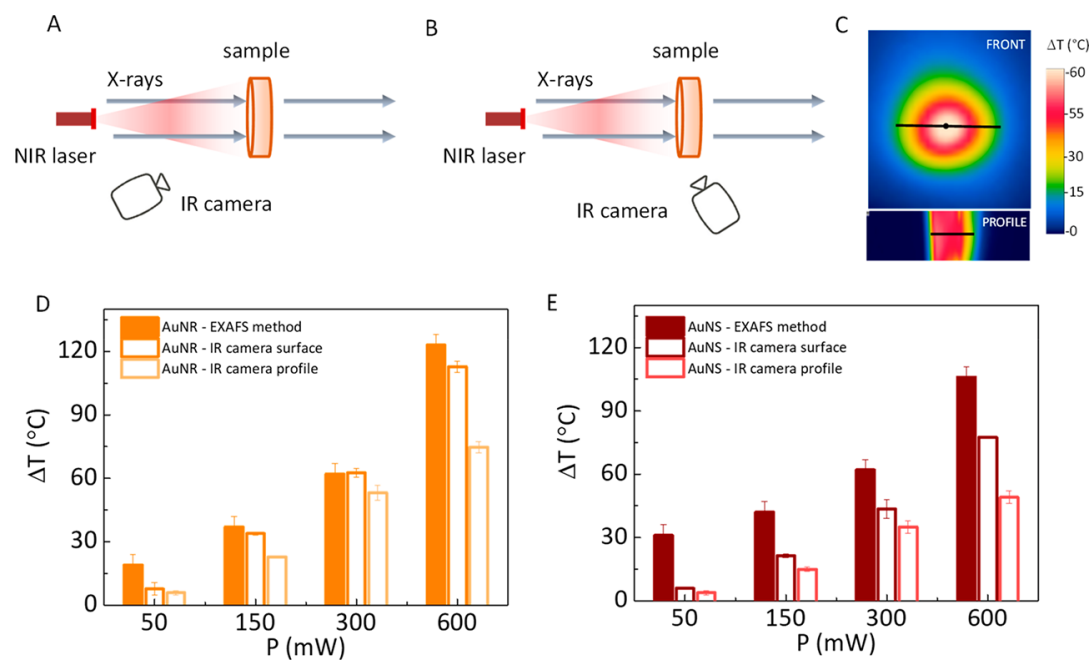

Figure 5. Macroscopic versus local temperature of single and hybrid gold-based nanomaterials under laser irradiation. Scheme of the two experimental configurations for measuring the macroscopic temperature of heat-excited Au based-nanoparticles using a thermographic camera pointed on the front surface (A) and along the profile view (B) of the sample. The sample is illuminated with an NIR light using a fiber-coupled laser at the same distance as the X-ray radiation experiment. (C) Infrared thermal image of AuNR after 15 min of laser irradiation at $808 \mathrm{~nm}$ and $0.6 \mathrm{~W}$. (D, E) Comparison of temperature increments for AuNR and AuNS subjected to different laser powers (reaching the thermal plateau) measured with the EXAFS technique and with the IR camera at both observation points.

ratio largely surpasses previous values at $0.05 \mathrm{~mW}$ laser illumination power (almost 10 times higher, $\Delta T=31{ }^{\circ} \mathrm{C}$ (EXAFS method) vs $\Delta T=6$ and $4{ }^{\circ} \mathrm{C}$ (global temperature)), indicating a subjacent minor heat dissipation into the matrix. This effect is attributable to the association of gold with the attached iron oxide component at each nanoparticle. The iron oxide lobe partially hampers the thermal diffusion of emanating photoinduced heat of gold nanoparticles to the surrounding matrix due to its low thermal conductance compared with that of metallic gold ( $2.5 \mathrm{vs} 310 \mathrm{~W} / \mathrm{mK}$ ). Besides, the temperature increment of AuNS is lower than the ones obtained with AuNR due to the inferior absorption at the selected $808 \mathrm{~nm}$ irradiation wavelength (see UV-vis-IR spectra in Figure 2). In both cases, photothermal capacity increases linearly with the density laser power.

These results evidence a nanoscopic local temperature significantly higher than global temperature measurements. This is in agreement with previous experimental and theoretical studies on nanoparticles subjected to either magneto- or photothermal heating modalities. For instance, the effect of MHT has been reported to induce a hightemperature gradient from the magnetic nanoparticle surface (from a few decimals (angstroms) to $5 \mathrm{~nm}$ distance), leading to localized heating 2-3 fold higher than the macroscopic environment, ${ }^{13,21,23}$ reaching temperature increments up to $40-80{ }^{\circ} \mathrm{C}$, depending on the distance from the nanoparticle vicinity. This confined heating allows release of a drug or synthesis/degradation of chemical species (as polymerization) more efficiently with magnetic stimulation than with macroscopic heating. ${ }^{41-43}$ In the case of photothermal excitation, it was shown by using temperature-dependent fluorescence nanolabels that gold-based nanomaterials undergo temperature distributions in their vicinity up to multiple folds higher than the global temperature upon laser heating of nanomaterials embedded either in liquid or solid matrix. ${ }^{12,14,44}$ These experiments based on temperature-dependent physical properties of nanothermometers do not yet provide the temperature of the heated nanoparticle itself, but rather the one of the thermometers situated close. In some cases, nanomaterials allowed dual NIR laser heaters and thermal sensors, such as rare earth emitters, ${ }^{45}$ quantum dots, or rotating plasmonic nanoparticles, ${ }^{24}$ with strong heating effects based on temperature-dependent fluorescence or other optical properties. Large thermal gradients have been then described using different nanosystems and techniques, revealing high temperature variations within a small volume. The determination of the local temperature of gold nanoparticles under NIR laser heating using X-ray absorption spectroscopy has been also studied by Broek and coworkers. ${ }^{46}$ Contrary to our results, they found no discrepancies between the macroscopic temperature (measured with an optical thermal probe) and the temperature of nanomaterials dispersed in aqueous solution. In our experiments, a solid matrix of partially hydrated $\mathrm{BN}$ was selected due to its transparency to both NIR light and X-rays, which is optimal for X-ray transmission detection due to its good signal-to-noise ratio. The results are able to be completely extrapolated to hyperthermic processes in a liquid matrix or in an equivalent physical environmental situation. The relatively lower specific heat $(1.6 \mathrm{~J} / \mathrm{gK})$ and higher thermal conductance $(125 \mathrm{~W} / \mathrm{mK})$ of $\mathrm{BN}$ than those for water $(4.18 \mathrm{~J} / \mathrm{gK}$ and $0.6 \mathrm{~W} / \mathrm{mK}$, respectively) make $\mathrm{BN}$ a better heat medium for heat dissipation. Therefore, the temperature gradients for the NPs would be even larger in an aqueous dispersion or cell conditions, and the difference of temperatures between the NPs and the medium could be even more pronounced, thus known as the "hot spot" effect. This methodology can be extended to any other applications that require the control of hot spot formations as plasmon-assisted catalytic reactions, ${ }^{47}$ nanoparticle-based water purification treatments, $^{48,49}$ or photovoltaic solar cells. ${ }^{50}$

In this work, we presented an in situ method to accurately determine the local temperature of nanomaterials. The validity of the method has been demonstrated in two types of different plasmonic gold-based nanomaterials, single and hybrid nanocrystals, used for biomedical applications. We provided an exhaustive study of heat transfer mechanisms and temperature reached at the heart of these nanomaterials subjected to NIR laser heating by using two temperature probe approaches 
working on different scales. Significant thermal gradients have been detected at the nanoscale by means of X-ray absorption spectroscopy as an onsite probe to correlate the local structural and vibrational thermal parameters of heated atoms. At low laser illumination power, this hot effect is highly pronounced. For hybrid gold nanomaterials combined with iron oxide, even larger temperature differences were observed. This nanothermometric method can be universally extended to any kind of nano-object under localized hyperthermic conditions in any environment and is applicable to monitor biological thermal events.

\section{ASSOCIATED CONTENT}

\section{(s) Supporting Information}

The Supporting Information is available free of charge at https://pubs.acs.org/doi/10.1021/acs.nanolett.0c04477.

\section{Additional figures (PDF)}

\section{AUTHOR INFORMATION}

\section{Corresponding Authors}

Ana Espinosa - IMDEA Nanociencia, 28049 Madrid, Spain; Nanobiotecnología (IMDEA-Nanociencia), Unidad Asociada al Centro Nacional de Biotecnología (CSIC), 28049 Madrid, Spain; (1) orcid.org/0000-0002-5626-6129;

Email: ana.espinosa@imdea.org

Álvaro Muñoz-Noval - Departamento de Física Materiales, Facultad CC. Físicas, Universidad Complutense de Madrid, 28040 Madrid, Spain; Email: almuno06@ucm.es

\section{Authors}

German R. Castro - Spanish CRG Beamline at the European Synchrotron (ESRF), F-38043 Grenoble, France; Instituto de Ciencia de Materiales de Madrid, ICMM-CSIC, 28049 Madrid, Spain

Javier Reguera - BCMaterials, Basque Center Centre for Materials, Applications and Nanostructures, 48940 Leioa, Spain; (1) orcid.org/0000-0001-5110-5361

Carlo Castellano - Dipartimento di Chimica, Università degli Studi di Milano, 20133 Milano, Italy

Javier Castillo - IMDEA Nanociencia, 28049 Madrid, Spain

Julio Camarero - IMDEA Nanociencia, 28049 Madrid, Spain; Departamento de Física de la Materia Condensada and Instituto 'Nicolás Cabrera', Universidad Autónoma de Madrid, 28049 Madrid, Spain

Claire Wilhelm - Laboratoire Matière et Systèmes, Complexes MSC, UMR 7057, CNRS \& University Paris Diderot, Paris Cedex 13 75205, France; 이잉.org/0000-0001-70249627

Miguel Angel García - Departamento de Electrocerámica, Instituto de Cerámica y Vidrio, ICV-CSIC, 28049 Madrid, Spain

Complete contact information is available at:

https://pubs.acs.org/10.1021/acs.nanolett.0c04477

\section{Notes}

The authors declare no competing financial interest.

\section{ACKNOWLEDGMENTS}

This work was supported by Comunidad de Madrid $\mathrm{I}+\mathrm{D}+\mathrm{i}$ program (Atracción de Talento project 2018-T1/IND-10360 (A.M-N.), Atracción de Talento project 2018-T1/IND-1005 (A.E.), and NANOMAGCOST project 2018/NMT-4321 and
MINECO project SEV-2016-0686. A.E. also acknowledges support from the Asociación Española contra el Cáncer program (AECC Ideas Semilla 2019). J.R. is thankful for funding from the Spanish State Research Agency (AEI) through the project PID2019-106099RB-C43/AEI/10.13039/ 501100011033. We acknowledge the Spanish National Research Council (CSIC) (PIE-2010-60-E013), the Spanish Ministry of Science and Innovation (MCINN), and the ESRF for financial support, provision of synchrotron radiation facilities, and BM25 personnel for technical support in using the beamline beyond their duties.

\section{REFERENCES}

(1) Jain, P. K.; Huang, X.; El-Sayed, I. H.; El-Sayed, M. A. Noble metals on the nanoscale: optical and photothermal properties and some applications in imaging, sensing, biology, and medicine. Acc. Chem. Res. 2008, 41 (12), 1578-1586.

(2) Rosensweig, R. E. Heating magnetic fluid with alternating magnetic field. J. Magn. Magn. Mater. 2002, 252, 370-374.

(3) Balfourier, A.; Mulens-Arias, V.; Gazeau, F.; Carn, F. Rational Design of Fractal Gold Nanosphere Assemblies with Optimized Photothermal Conversion Using a Quantitative Structure Property Relationship (QSPR) Approach. J. Phys. Chem. C 2020, 124 (16), 8938-8948.

(4) Serantes, D.; Simeonidis, K.; Angelakeris, M.; ChubykaloFesenko, O.; Marciello, M.; Morales, M. d. P.; Baldomir, D.; Martinez-Boubeta, C. Multiplying Magnetic Hyperthermia Response by Nanoparticle Assembling. J. Phys. Chem. C 2014, 118 (11), 59275934.

(5) Espinosa, A.; Curcio, A.; Cabana, S.; Radtke, G.; Bugnet, M.; Kolosnjaj-Tabi, J.; Péchoux, C.; Alvarez-Lorenzo, C.; Botton, G. A.; Silva, A. Intracellular Biodegradation of Ag Nanoparticles, Storage in Ferritin, and Protection by $\mathrm{Au}$ Shell for Enhanced Photothermal Therapy. ACS Nano 2018, 12, 6523.

(6) Espinosa, A.; Kolosnjaj-Tabi, J.; Abou-Hassan, A.; Plan Sangnier, A.; Curcio, A.; Silva, A. K.; Di Corato, R.; Neveu, S.; Pellegrino, T.; Liz-Marzán, L. M. Magnetic (Hyper) Thermia or Photothermia? Progressive Comparison of Iron Oxide and Gold Nanoparticles Heating in Water, in Cells, and In Vivo. Adv. Funct. Mater. 2018, 28 (37), 1803660.

(7) Chen, R.; Romero, G.; Christiansen, M. G.; Mohr, A.; Anikeeva, P. Wireless magnetothermal deep brain stimulation. Science 2015, 347 (6229), 1477-1480.

(8) Stanley, S. A.; Gagner, J. E.; Damanpour, S.; Yoshida, M.; Dordick, J. S.; Friedman, J. M. Radio-wave heating of iron oxide nanoparticles can regulate plasma glucose in mice. Science 2012, 336 (6081), 604-608.

(9) Zhou, J.; Del Rosal, B.; Jaque, D.; Uchiyama, S.; Jin, D. Advances and challenges for fluorescence nanothermometry. Nat. Methods 2020, 17, 1-14.

(10) Ruiz, D.; del Rosal, B.; Acebrón, M.; Palencia, C.; Sun, C.; Cabanillas-González, J.; López-Haro, M.; Hungría, A. B.; Jaque, D.; Juarez, B. H. Ag/Ag2S Nanocrystals for High Sensitivity NearInfrared Luminescence Nanothermometry. Adv. Funct. Mater. 2017, 27 (6), 1604629.

(11) Qiu, X.; Zhou, Q.; Zhu, X.; Wu, Z.; Feng, W.; Li, F. Ratiometric upconversion nanothermometry with dual emission at the same wavelength decoded via a time-resolved technique. Nat. Commun. 2020, 11 (1), 1-9.

(12) Rohani, S.; Quintanilla, M.; Tuccio, S.; De Angelis, F.; Cantelar, E.; Govorov, A. O.; Razzari, L.; Vetrone, F. Enhanced Luminescence, Collective Heating, and Nanothermometry in an Ensemble System Composed of Lanthanide-Doped Upconverting Nanoparticles and Gold Nanorods. Adv. Opt. Mater. 2015, 3 (11), 1606-1613.

(13) Dong, J.; Zink, J. I. Taking the temperature of the interiors of magnetically heated nanoparticles. ACS Nano 2014, 8 (5), 51995207. 
(14) Debasu, M. L.; Ananias, D.; Pastoriza-Santos, I.; Liz-Marzán, L. M.; Rocha, J.; Carlos, L. D. All-in-one optical heater-thermometer nanoplatform operative from 300 to $2000 \mathrm{~K}$ based on Er3+ emission and blackbody radiation. Adv. Mater. 2013, 25 (35), 4868-4874.

(15) Ximendes, E. C.; Pereira, A. F.; Rocha, U.; Silva, W. F.; Jaque, D.; Jacinto, C. Thulium doped LaF 3 for nanothermometry operating over $1000 \mathrm{~nm}$. Nanoscale 2019, 11 (18), 8864-8869.

(16) Rocha, U.; Hu, J.; Rodríguez, E. M.; Vanetsev, A. S.; Rähn, M.; Sammelselg, V.; Orlovskii, Y. V.; Solé, J. G.; Jaque, D.; Ortgies, D. H. Subtissue Imaging and Thermal Monitoring of Gold Nanorods through Joined Encapsulation with Nd-Doped Infrared-Emitting Nanoparticles. Small 2016, 12 (39), 5394-5400.

(17) Ximendes, E. C.; Santos, W. Q.; Rocha, U. s.; Kagola, U. K.; Sanz-Rodríguez, F.; Fernández, N.; Gouveia-Neto, A. d. S.; Bravo, D.; Domingo, A. M.; del Rosal, B. Unveiling in vivo subcutaneous thermal dynamics by infrared luminescent nanothermometers. Nano Lett. 2016, 16 (3), 1695-1703.

(18) Ortgies, D. H.; Teran, F. J.; Rocha, U.; de la Cueva, L.; Salas, G.; Cabrera, D.; Vanetsev, A. S.; Rähn, M.; Sammelselg, V.; Orlovskii, Y. V. Optomagnetic nanoplatforms for in situ controlled hyperthermia. Adv. Funct. Mater. 2018, 28 (11), 1704434.

(19) Hemmer, E.; Quintanilla, M.; Legare, F.; Vetrone, F. Temperature-induced energy transfer in dye-conjugated upconverting nanoparticles: a new candidate for nanothermometry. Chem. Mater. 2015, 27 (1), 235-244.

(20) Piñol, R.; Zeler, J.; Brites, C. D.; Gu, Y.; Téllez, P.; Carneiro Neto, A. N.; da Silva, T. E.; Moreno-Loshuertos, R.; Fernandez-Silva, P.; Gallego, A. I. Real-time intracellular temperature imaging using lanthanide-bearing polymeric micelles. Nano Lett. 2020, 20 (9), 6466-6472.

(21) Riedinger, A.; Guardia, P.; Curcio, A.; Garcia, M. A.; Cingolani, R.; Manna, L.; Pellegrino, T. Subnanometer local temperature probing and remotely controlled drug release based on azofunctionalized iron oxide nanoparticles. Nano Lett. 2013, 13 (6), 2399-2406.

(22) Polo-Corrales, L.; Rinaldi, C. Monitoring iron oxide nanoparticle surface temperature in an alternating magnetic field using thermoresponsive fluorescent polymers. J. Appl. Phys. 2012, 111 (7), No. 07 B334.

(23) Dias, J. T.; Moros, M.; del Pino, P.; Rivera, S.; Grazú, V.; de la Fuente, J. M. DNA as a molecular local thermal probe for the analysis of magnetic hyperthermia. Angew. Chem., Int. Ed. 2013, 52 (44), 11526-11529.

(24) Maity, S.; Wu, W.-C.; Tracy, J. B.; Clarke, L. I.; Bochinski, J. R. Nanoscale steady-state temperature gradients within polymer nanocomposites undergoing continuous-wave photothermal heating from gold nanorods. Nanoscale 2017, 9 (32), 11605-11618.

(25) Jaque, D.; Maestro, L. M.; Del Rosal, B.; Haro-Gonzalez, P.; Benayas, A.; Plaza, J.; Rodriguez, E. M.; Sole, J. G. Nanoparticles for photothermal therapies. Nanoscale 2014, 6 (16), 9494-9530.

(26) Kelly, K. L.; Coronado, E.; Zhao, L. L.; Schatz, G. C. The optical properties of metal nanoparticles: the influence of size, shape, and dielectric environment. J. Phys. Chem. B 2003, 107, 668-677.

(27) Jain, P. K.; Lee, K. S.; El-Sayed, I. H.; El-Sayed, M. A. Calculated absorption and scattering properties of gold nanoparticles of different size, shape, and composition: applications in biological imaging and biomedicine. J. Phys. Chem. B 2006, 110 (14), 72387248.

(28) Chen, G. Nonlocal and nonequilibrium heat conduction in the vicinity of nanoparticles. J. Heat Transfer 1996, 118, 539-545.

(29) Chen, G. Ballistic-diffusive heat-conduction equations. Phys. Rev. Lett. 2001, 86 (11), 2297.

(30) Vaccari, M.; Fornasini, P. Einstein and Debye models for EXAFS parallel and perpendicular mean-square relative displacements. J. Synchrotron Radiat. 2006, 13 (4), 321-325.

(31) Beni, G.; Platzman, P. Temperature and polarization dependence of extended x-ray absorption fine-structure spectra. Phys. Rev. B 1976, 14 (4), 1514.
(32) Shafai, G.; Ortigoza, M. A.; Rahman, T. S. Vibrations of Au13 and FeAu12 nanoparticles and the limits of the Debye temperature concept. J. Phys.: Condens. Matter 2012, 24 (10), 104026.

(33) Purans, J.; Timoshenko, J.; Kuzmin, A.; Dalba, G.; Fornasini, P.; Grisenti, R.; Afify, N.; Rocca, F.; De Panfilis, S.; Ozhogin, I. Femtometer accuracy EXAFS measurements: Isotopic effect in the first, second and third coordination shells of germanium. Journal of Physics: Conference Series 2009, 190 (1), No. 012063.

(34) Pettifer, R. F.; Mathon, O.; Pascarelli, S.; Cooke, M. D.; Gibbs, $\mathrm{M}$. R. Measurement of femtometre-scale atomic displacements by $\mathrm{X}$ ray absorption spectroscopy. Nature 2005, 435 (7038), 78.

(35) Araujo, L.; Kluth, P.; Azevedo, G. D. M.; Ridgway, M. C. Vibrational properties of Ge nanocrystals determined by EXAFS. Phys. Rev. B: Condens. Matter Mater. Phys. 2006, 74 (18), 184102.

(36) Espinosa, A.; Reguera, J.; Curcio, A.; Muñoz-Noval, A.; Kuttner, C.; Van de Walle, A.; Liz-Marzán, L. M.; Wilhelm, C. Janus Magnetic-Plasmonic Nanoparticles for Magnetically Guided and Thermally Activated Cancer Therapy. Small 2020, 16, 1904960.

(37) Reguera, J.; de Aberasturi, D. J.; Winckelmans, N.; Langer, J.; Bals, S.; Liz-Marzán, L. M. Synthesis of Janus plasmonic-magnetic, star-sphere nanoparticles, and their application in SERS detection. Faraday Discuss. 2016, 191, 47-59.

(38) Chevrier, D.; Chatt, A.; Sham, T.; Zhang, P. A Comparative XAFS Study of Gold-thiolate Nanoparticles and Nanoclusters. J. Phys.: Conf. Ser. 2013, 430 (1), No. 012029.

(39) Duan, Z.; Li, Y.; Timoshenko, J.; Chill, S. T.; Anderson, R. M.; Yancey, D. F.; Frenkel, A. I.; Crooks, R. M.; Henkelman, G. A combined theoretical and experimental EXAFS study of the structure and dynamics of Au 147 nanoparticles. Catal. Sci. Technol. 2016, 6 (18), 6879-6885.

(40) Espinosa, A.; Di Corato, R.; Kolosnjaj-Tabi, J.; Flaud, P.; Pellegrino, T.; Wilhelm, C. Duality of iron oxide nanoparticles in cancer therapy: amplification of heating efficiency by magnetic hyperthermia and photothermal bimodal treatment. ACS Nano 2016, 10 (2), 2436-2446.

(41) Cazares-Cortes, E.; Espinosa, A.; Guigner, J.-M.; Michel, A.; Griffete, N.; Wilhelm, C.; Ménager, C. Doxorubicin intracellular remote release from biocompatible oligo (ethylene glycol) methyl ether methacrylate-based magnetic Nanogels triggered by magnetic hyperthermia. ACS Appl. Mater. Interfaces 2017, 9 (31), 2577525788.

(42) Griffete, N.; Fresnais, J.; Espinosa, A.; Taverna, D.; Wilhelm, C.; Ménager, C. Thermal polymerization on the surface of iron oxide nanoparticles mediated by magnetic hyperthermia: Implications for multishell grafting and environmental applications. ACS Applied Nano Materials 2018, 1 (2), 547-555.

(43) Urraca, J. L.; Cortés-Llanos, B. n.; Aroca, C.; Presa, P. d. 1.; Pérez, L.; Moreno-Bondi, M. C. Magnetic field-induced polymerization of molecularly imprinted polymers. J. Phys. Chem. C 2018, 122 (18), 10189-10196.

(44) Nigoghossian, K.; Ouellet, S.; Plain, J.; Messaddeq, Y.; Boudreau, D.; Ribeiro, S. J. Upconversion nanoparticle-decorated gold nanoshells for near-infrared induced heating and thermometry. $J$. Mater. Chem. B 2017, 5 (34), 7109-7117.

(45) Ximendes, E. C.; Rocha, U.; Kumar, K. U.; Jacinto, C.; Jaque, D. LaF3 core/shell nanoparticles for subcutaneous heating and thermal sensing in the second biological-window. Appl. Phys. Lett. 2016, 108 (25), 253103.

(46) Van de Broek, B.; Grandjean, D.; Trekker, J.; Ye, J.; Verstreken, K.; Maes, G.; Borghs, G.; Nikitenko, S.; Lagae, L.; Bartic, C. Temperature Determination of Resonantly Excited Plasmonic Branched Gold Nanoparticles by X-ray Absorption Spectroscopy. Small 2011, 7 (17), 2498-2506.

(47) Ren, X.; Cao, E.; Lin, W.; Song, Y.; Liang, W.; Wang, J. Recent advances in surface plasmon-driven catalytic reactions. RSC Adv. 2017, 7 (50), 31189-31203.

(48) Wei, H.; Loeb, S. K.; Halas, N. J.; Kim, J.-H. Plasmon-enabled degradation of organic micropollutants in water by visible-light 
illumination of Janus gold nanorods. Proc. Natl. Acad. Sci. U. S. A.

2020, 117 (27), 15473-15481.

(49) Dongare, P. D.; Alabastri, A.; Pedersen, S.; Zodrow, K. R.; Hogan, N. J.; Neumann, O.; Wu, J.; Wang, T.; Deshmukh, A.; Elimelech, M. Nanophotonics-enabled solar membrane distillation for off-grid water purification. Proc. Natl. Acad. Sci. U. S. A. 2017, 114 (27), 6936-6941.

(50) Kuppe, C.; Rusimova, K. R.; Ohnoutek, L.; Slavov, D.; Valev, V. K. Hot" in Plasmonics: Temperature-Related Concepts and Applications of Metal Nanostructures. Adv. Opt. Mater. 2020, 8 (1), 1901166. 